\title{
Cuestionario de Riesgo de AUtolesión (CRA): Propiedades PSICOMÉtricas Y RESULTADOS EN UNA MUESTRA DE ADOLESCENTES
}

\author{
Modesto Solis Espinoza y Gilda Gómez-Peresmitré \\ Universidad Nacional Autónoma de México \\ México
}

\begin{abstract}
RESUMEN
La autolesión tiene una alta frecuencia en adolescentes, por lo que son necesarios instrumentos adecuados para su medición y acciones preventivas. El objetivo de este estudio fue elaborar un instrumento válido y confiable que explorara la presencia de autolesión y aspectos muy poco abordados, como dificultad para dejar de lesionarse y el factor de riesgo denominado contagio social. De una muestra total $(N=629)$ no probabilística se obtuvo una muestra con distribución normal con $N=241$ estudiantes de secundarias públicas del sur de la Ciudad de México, 89 hombres y 152 mujeres, con $M=12.37$ años $(D E=2.28)$ y rango de 11-15 años. Los ítems del instrumento se elaboraron con base en la literatura y se sometieron a procedimientos de validación de contenido (evaluación de expertos), laboratorios cognoscitivos (para asegurar comprensión/significado de los ítems), validez de constructo, análisis factorial exploratorio (AFE) y confirmatorio (AFC), y consistencia interna (coeficientes alpha y omega). El AFC proyectó un modelo con tres factores (frecuencia, efecto adictivo y contagio social) con índices de bondad de ajuste adecuados y consistencia interna aceptable (alpha y omega $=0.94$ ). Los resultados indican que el cuestionario es útil para evaluar aspectos relevantes de la autolesión; se discute la necesidad de complementar la información con entrevistas y de ahondar en el contagio social y la intención de autolesionarse.
\end{abstract}

Palabras Clave:

autolesión, adolescentes, contagio social, análisis factorial confirmatorio, validez de constructo

\section{SELF-HARM RISK (CRA) QUESTIONNAIRE: PSYCHOMETRIC PROPERTIES AND RESULTS IN A SAMPLE OF ADOLESCENTS}

\begin{abstract}
Self-injury has a high frequency in adolescents, so appropriate instruments are required for its measurement and preventive actions. The purpose of this study was to develop a valid and reliable instrument that explores the presence of self-injury and aspects very little addressed, such as difficulty to stop injuring oneself and the risk factor called social contagion. From a non-probabilistic total sample $(\mathrm{N}=629)$, a sample with a normal distribution was obtained with $\mathrm{N}=241$ public secondary students from southern Mexico City, 89 men and 152 women ( $M=12.37$ years, $S D=2.28$, range 11-15 years) The items of the instrument were constructed based on the literature and subjected to content validation procedures (expert evaluation), cognitive laboratories (to ensure understanding/meaning of the items), construct validity, exploratory factorial analysis (EFA) and confirmatory (CFA) and internal consistency (alpha and omega coefficients). The CFA yielded a model with three factors (frequency, addictive effect and social contagion) with adequate goodness of fit indexes and acceptable internal consistency (alpha and omega $=0.94$ ). The results indicate that the questionnaire is useful to evaluate relevant aspects of the self-injury, it is duscussed the needed to complement the information with interviews, to focus on social contagion and the intention of sel-finjury.
\end{abstract}

Keywords:

self-injury, adolescents, social contagion, construct validity, confirmatory factor analysis

Bitácora del Artículo:

| Recibido: 11 de Abril de 2019 | Aceptado: 20 de Septiembre de 2019 | Publicado en línea: Enero - Junio de 2020 | 
Artículo Empírico | Cuestionario de riesgo de autolesión...| Solis-Espinoza a L Gómez-Peresmitré

Autoría y Derechos de Propiedad Intelectual

Cuestionario de RIESgo de aUtolesión (CRA): PROPIEDAdes PSICOMÉTRICAS Y RESULTADOS EN UNA MUESTRA DE ADOLESCENTES

\author{
Modesto Solis Espinoza y Gilda Gómez-Peresmitré \\ Universidad Nacional Autónoma de México \\ México
}

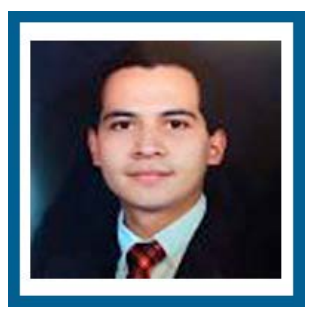

Modesto Solís Espinoza

Facultad de Psicología, UNAM

Correo: modesto333_3@hotmail.com

Cursó la carrera de Psicología en la Facultad de Psicología de la UNAM con un semestre de intercambio académico en la Universidad Piloto de Colombia, actualmente postulante a doctor en Psicología y Salud en la UNAM.

\title{
CONTRIBUCIÓN DE LOS AUTORES
}

Modesto Solís Espinoza: Participación en todo el proceso de elaboración, desarrollo del proyecto, aplicación de instrumentos y entrevistas, análisis de datos y redacción del artículo. | Gilda Gómez-Peresmitre: Supervisión del desarrollo del proyecto y corrección de estilo en la redacción del instrumento.

\section{AGRADECIMIENTOS}

Se agradece al Consejo Nacional de Ciencia y Tecnología el apoyo para realizar la presente investigación.

\section{DAtos de Filiación de los Autores}

Facultad de Psicología, UNAM.

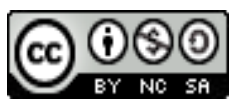

Copyright: (C) 2020 Solís-Espinoza, M. \& Gómez-Peresmitre, G.

Este es un artículo de acceso abierto distribuido bajo los términos de la licencia Creative Commons Reconocimiento-NoComercial 4.0 Internacional, por lo que su contenido gráfico y escrito se puede compartir, copiar y redistribuir total o parcialmente sin necesidad de permiso expreso de sus autoras con la única condición de que no se puede usar con fines directamente comerciales y los términos legales de cualquier trabajo derivado deben ser los mismos que se expresan en la presente declaración. La única condición es que se cite la fuente con referencia a la Revista Digital Internacional de Psicología y Ciencia Social y a sus autoras. 


\section{TABLA DE CONTENIDO}

MÉTODO

112

Participantes, 112

Materiales, 113

Procedimiento, 113

Análisis estadísticos, 113

\section{RESULTADOS}

Validez de contenido, 113

Análisis factorial exploratorio (AFE), 113

Análisis factorial confirmatorio (AFC), 114

\section{Discusión}

CONCLUSIONES

REFERENCIAS 
$\mathbf{L}$ a autolesión es un acto intencional y autoinflingido; conlleva un daño corporal de baja letalidad, de nadectura socialmente inaceptable; tiene como finalidad reducir el dolor emocional (Walsh, 2006), se efectúa sin intencionalidad suicida consciente y la mayoría de las veces sucede en ausencia de psicosis y/o impedimentos intelectuales; en tales casos se le conoce como autolesión psicótica (presente en trastornos graves como esquizofrenia y en episodios de psicosis como consecuencia de la pérdida de contacto con la realidad) y autolesión orgánica (presente en retraso mental y autismo con la función de autoestimulación) (Favazza, 1996; Mangall \& Yurkovich, 2008; Santos, 2011).

En revisiones sistemáticas se ha encontrado una prevalencia que oscila entre 7.5 y $46.5 \%$ en adolescentes, siendo las edades entre 12 y 14 años en que más incidencia se ha reportado (Cipriano, Cella \& Cotrufo, 2017). En otros estudios se indica que la prevalencia en muestras comunitarias de adolescentes es de $21 \%$ (Gandhi, Luyckx, Baetens, Kiekens, Sleuwaegen, Berens y Claes, 2017). En México. dependiendo de los criterios utilizados para la detección de autolesión, se ha identificado una prevalencia de entre 5.6 a $17.1 \%$ (Albores-Gallo, Méndez-Santos, García, Delgadillo-González, Chávez y Martínez, 2014), cifras alarmantes porque se considera que hasta $50 \%$ de los que se producen autolesión pueden padecer un trastorno mental (Farber, Jackson, Tabin \& Bachar, 2007) y pueden estar relacionados de modo indirecto con otros comportamientos autodestructivos, como el abuso de sustancias adictivas y de ayunos (Pattison \& Kahan, 1983).

Puede decirse que no obstante el impacto que puede tener la autolesión en el desarrollo de los adolescentes todavía no existe consenso respecto a su definición y evaluación. Hasta hace pocos años aún se le conceptualizaba como síntoma del trastorno límite de personalidad (TLP), tratándola, por tanto, como parte de dicho trastorno o adjudicándole otras características derivadas de esa asociación. Sin embargo, a partir de la alta prevalencia de conductas autolesivas en ausencia de TLP surgió la propuesta en el DSM 5 (American Psychiatric Association, 2013) de definir la autolesión no suicida como trastorno, lo que permite mejorar el tratamiento y sistematizando o estandarizando la investigación respecto a la misma (Albores-Gallo et al., 2014; Eisenkraft, 2006).
Son escasos los instrumentos que evalúan autolesión en población hispanohablante, y de los existentes hay pocas evidencias de su validez y confiabilidad (Flores, Hidalgo-Rasmussen \& Peñúñuri, 2017). En adolescentes mexicanos se ha empleado por ejemplo la cédula de autolesión (Marín, 2013); sin embargo, sin reporte de las propiedades psicométricas; de igual manera se ha utilizado el Inventario de autolesión deliberada (Deliberate Self-Harm Inventory, DSHI) (Gratz, 2001), pero también se carece de información relacionada con el proceso de validación y confiabilización. Otro instrumento utilizado es el Self-Harm Questionnaire (cuestionario de autolesión), que sí tiene propiedades psicométricas adecuadas, además de explorar otras áreas, como la afectiva y el consumo de sustancias. También se ha usado la Cédula de autolesiones (Albores-Gallo et al., 2014), que sí tiene propiedades psicométricas reportadas y abarca varios aspectos de la autolesión, explorando incluso las partes del cuerpo donde éstas se efectúan. Por otra parte se debe considerar la razón costo/beneficio de incluir en los cuestionarios, catálogos de las diversas formas de autolesión, como sucede con algunos de los instrumentos señalados, para evitar promover o incitar a los jóvenes a explorar más o nuevos modos de autolesión.

Con base en lo mencionado y dada la necesidad de tener con más información que permita diseñar mejores programas de prevención, se planteó como propósito de investigación desarrollar un instrumento válido y confiable que explorara otros aspectos no abordados por los instrumentos ya existentes, tales como la intención de iniciar la conducta de autolesión, el efecto adictivo o dificultad para dejar de hacerlo, así como el efecto de riesgo del contagio social —observar y conocer otras personas que lo hacen-. También se propone conocer la frecuencia con que se efectúa la autolesión para deteminar su prevalencia como factor de riesgo, porque el criterio propuesto por el DSM 5 (autolesión durante cinco días en el último año) (APA, 2013) se aplica para el diagnóstico de la patología.

\section{MÉTOdo}

\section{Participantes}

Se obtuvo una muestra total no probabilística de $N=629$ adolescentes; se descartaron los casos en que no se reportó autolesión ni intención de efectuarla, quedando una $\mathrm{n}$ = 241 participantes (89 hombres y 152 mujeres), con $\mathrm{M}=$ 12.37 años, $(\mathrm{DE}=2.28)$ y rango de 11-15 años. 


\section{Materiales}

Se elaboró un cuestionario de riesgo de autolesión (CRA) con una sección de datos sociodemográficos, como edad y sexo, y preguntas relacionadas con la conducta de autolesión, tales como motivo, modo y edad en que se inició la misma, y otra sección con 16 ítems tanto dicotómicos como politómicos. explorando presencia de autolesión, intención de hacerla, frecuencia de la misma, dificultad para dejar de herirse y contagio social. Los items se establecieron con base en la literatura, y se sometieron a procedimientos de validación de contenido (evaluación de expertos), laboratorios cognoscitivos (para asegurar comprensión/significado de los ítems), validez de constructo (análisis factoriales) y consistencia interna (coeficientes alpha y omega). Se desarrolló el protocolo de una entrevista semiestructurada con el propósito de revisar concordancia entre respuestas cuestionario/entrevista.

\section{Procedimiento}

Se solicitaron a los directivos de tres secundarias públicas ubicadas al sur de la Ciudad de México permisos para efectuar el proyecto. Una vez conseguidos dichos permisos se procedió a la aplicación del cuestionario a los estudiantes en las aulas en los horarios de clase. Se les informó acerca de los propósitos de investigación, se hizo entrega de un consentimiento informado y la aplicación del instrumento duró casi una hora. Al final se entrevistó a $20 \%$ de los participantes, elegidos de modo aleatorio de entre quienes reportaron lesionarse a sí mismos. La recolección de datos fue de carácter no invasivo y se respetaron los criterios éticos de la investigación en Psicología (SMP, 2007). Para asegurar una comprensión adecuada del instrumento, se efectuaron dos laboratorios cognoscitivos, con ocho adolescentes de primero y segundo grados cada uno; los estudiantes tenían que leer el cuestionario y expresar lo que habían entendido en cada pregunta. Se procedió a obtener la validez por jueceo con cuatro expertos (psicólogos clínicos especializados en atención de adolescentes y con experiencia en el tratamiento de la autolesión); se les explicó la finalidad del instrumento y se les solicitó calificar como adecuado/inadecuado cada uno de los ítems en cuanto claridad, coherencia y relevancia, y se les pidieron observaciones respecto al orden y opciones de respuesta; se calculó el índice de concordancia interjueces con el coeficiente kappa de Fleiss (1971).

\section{Análisis estadísticos}

Para analizar las diferencias por sexo se hicieron pruebas $\mathrm{t}$ de Student para grupos independientes. El instrumento tiene ítems con dos y hasta siete opciones de respues- ta; sin embargo, para evitar estimaciones inadecuadas se igualaron todas las variables a un nivel de medición dicotómico, y de esta manera se efectuaron los análisis posteriores. Para la obtención de la validez de constructo y conocer la estructura factorial del instrumento se utilizó el análisis factorial exploratorio (AFE) a partir de una matriz de correlación tetracórica (Lloret-Segura, Ferreres-Traver, Hernández-Baeza \& Tomás-Marco, 2014); con el método de mínimos cuadrados no ponderados (ULS) y con rotación PROMIN (Lorenzo-Seva, 1999) se estimó el estadístico de adecuación de la muestra de Kaiser-Meyer-Olkin $(\mathrm{KMO})$ y la prueba de esfericidad. De acuerdo con los criterios convencionales los valores adecuados son: $\mathrm{KMO}$ $\geq 0.7$ (Kaiser, 1974) y prueba de esfericidad (X2 $p \leq 0.05$ ) (Bartlett, 1950); Factor $\geq 3$ ítems (Kim \& Mueller, 1978; Costello \& Osborne, 2005); ítems con cargas factoriales $\geq$ 0.40 (MacCallum, Widaman, Zhang \& Hong, 1999; Velicer y Fava, 1998; Williams, Brown y Onsman, 2010). Coeficientes de consistencia interna alphas de Cronbach $\geq 0.7$ (Nunnally, 1978). El análisis factorial confirmatorio (AFC) se hizo con el método de máxima verosimilitud (Satorra y Bentler, 1988; 1994). Con criterios e índices de bondad de ajuste: $\mathrm{X}^{2} / \mathrm{gl} \leq 3, \mathrm{CFI} \geq 0.90, \mathrm{GFI} \geq 0.90$, IFI $\geq 0.90$ (Hu y Bentler, 1999; Kline, 2005), RMSEA $\leq 0.07$ (Steiger, 2007). Se calculó el coeficiente alpha ordinal y omega para cada factor. Para tales propósitos se utilizó el paquete estadístico para las ciencias sociales (SPSS) v. 16, el paquete FACTOR v. 10.8.04 (Lorenzo-Seva y Ferrando, 2007), y el programa EQS 6.2.

\section{Resultados}

\section{Validez de contenido}

El índice total de concordancia kappa de Fleiss, con 95\% de intervalo de confianza resultó aceptable en cuanto a claridad (kappa $=0.75)$, coherencia $($ kappa $=0.78)$ y relevancia ( $k a p p a=0.86$ ). A partir de la evaluación por jueceo se modificó la redacción de algunos ítems y/o se cambiaron palabras para hacerlo más comprensible. La mayoría de las respuestas obtenidas en la entrevista $(90 \%)$ fueron consistentes con las respuestas dadas al cuestionario; lo contestado en entrevista coincidió con lo respondido en el instrumento; sin embargo hubo un bajo índice $(20 \%)$ de respuestas contradictorias, en especial respecto a frecuencia de autolesiones y la última vez en que se hicieron.

\section{Análisis factorial exploratorio (AFE)}

Antes de efectuar el AFE se estimó la medida de adecuación muestral de Kaiser-Meyer-Olkin, la cual dio un 
valor aceptable $(\mathrm{KMO}=0.82)$ y se aplicó a los datos la prueba de esfericidad de Bartlett, la cual resultó significativa $\left.X^{2}(120)=1133.8 ; p=0.001\right)$.

El AFE arrojó tres factores que agruparon 12 de los ítems; el alfa ordinal total fue de 0.94. El primer factor, frecuencia de autolesión (episodios de autolesión en diferentes periodos), se compone de cuatro ítems; explicó $31.26 \%$ de la varianza, con alpha ordinal $=0.89$. El segundo explora el efecto adictivo de la autolesión (dificultad para evitar lastimarse); consta de cinco ítems; explicó $14.37 \%$ de varianza, con alpha ordinal $=0.85$. El tercer factor explora el contagio social de la autolesión (observación y sugerencia de herirse); consta de tres ítems; explicó $9.48 \%$ de la varianza, con alpha ordinal $=0.76$. Las cargas factoriales de los 12 ítems fluctuaron entre 0.48 y 0.99. Los ítems correspondientes a la planeación o intención de autolesionarse no se mantuvieron dentro de la estructura factorial obtenida (tabla 1).

Tabla 1.

Análisis factorial exploratorio de CRA.

FACTOR $\begin{gathered}\text { CARGA } \\ \text { FACTORIAL }\end{gathered}$

Frecuencia ( $\alpha$ ordinal $=0.89 \Omega=0.89$ )
4. ¿ Cuándo fue la última vez que lo hiciste?
0.725
5. ¿Qué tan seguido te has lastimado en el último mes?
0.827
6. ¿Qué tan seguido te has lastimado en los últimos seis meses?

\subsection{8}
7. ¿Qué tan seguido te has lastimado en el último año?
0.858

Componente adictivo ( $\alpha$ ordinal $=0.85 \Omega=0.85$ )

\section{3 ¿Alguna vez te has lastimado apropósito? $\quad 0.687$ \\ 9 ¿Cuándo te has lastimado lo haz hecho con \\ la intención de quitarte la vida? \\ 0.601}

10 ¿Qué tan probable es que te vuelvas a lastimar?

0.818

11 ¿Qué tanto quisieras volver a lastimarte?

0.820

12 ¿Qué tan dificil es dejar de lastimarte?

0.734

Contagio social ( $\alpha$ ordinal $=0.76 \Omega=0.79$ )

13 ¿Antes de lastimarte te enteraste que alguien más lo hiciera?

0.712

14 ¿Antes de lastimarte viste que alguien lo hiciera?

15 ¿Alguien te sugierio lastimarte?

0.487

Consistencia interna

$\Omega$ total: 94

a total: 94

\section{Análisis factorial confirmatorio (AFC)}

Se procedió a probar la estructura de tres factores resultado del AFE; después de ensayar varios ajustes a las soluciones factoriales, el AFC produjo un modelo con tres factores que explicaron $55.12 \%$ de la varianza total; considerando un total de 12 ítems, se mantuvieron los mismos que en el AFE.

En la figura 1 se observa que el modelo final quedó conformado por tres factores correlacionados entre sí: frecuencia con efecto adictivo (0.714), este último con contagio social (.305), y contagio social con frecuencia (0.061), con un coeficiente de determinación o tamaño del efecto $\mathrm{R} 2=0.473$. Se mostró un buen ajuste del modelo considerando los siguientes índices: $\mathrm{X}^{2} / \mathrm{gl}=2.05, \mathrm{p}=0.000 ; \mathrm{GFI}=$ 0.93; $\mathrm{IFI}=0.94 ; \mathrm{CFI}=0.94 ; \mathrm{RMSEA}=0.066$.

\begin{tabular}{|l|l|}
\hline \multicolumn{2}{|c|}{ AFC } \\
\hline $\mathrm{X}^{2} / \mathrm{gl}$ & 2.05 \\
\hline RMSEA & .066 \\
\hline CFI & .946 \\
\hline GFI & .937 \\
\hline IFI & .947 \\
\hline R $^{2}$ & .473 \\
\hline
\end{tabular}

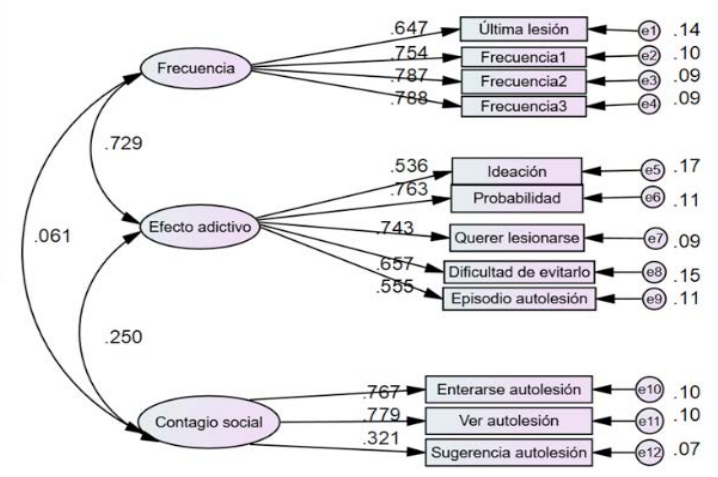

Figura 1.

Modelo del CRA

\section{Prevalencia de autolesión}

De la muestra total ( $N=629), 185(29.41 \%)$ adolescentes afirmaron haber tenido al menos un episodio de autolesión; 72 hombres (11.44\%) y 113 mujeres (17.96\%); en la tabla 2 se muestra el análisis de la frecuencia con que se tuvieron episodios de autolesión en diferentes lapsos.

Tabla 2.

Prevalencia de autolesión.

\begin{tabular}{|c|c|c|c|}
\hline Frecuencia & TOTAL & HOMBres & Mujeres \\
\hline \multicolumn{4}{|c|}{ Última autolesión } \\
\hline Hoy & $1.62 \%$ & $0.5 \%$ & $1.08 \%$ \\
\hline Esta semana & $10.27 \%$ & $4.3 \%$ & $5.94 \%$ \\
\hline Este mes & $19.45 \%$ & $7.56 \%$ & $11.89 \%$ \\
\hline $\begin{array}{l}\text { Hace seis } \\
\text { meses }\end{array}$ & $7 \%$ & $1.62 \%$ & $5.4 \%$ \\
\hline Este año & $12.43 \%$ & $3.24 \%$ & $9.18 \%$ \\
\hline
\end{tabular}




\begin{tabular}{|lccc|}
\hline $\begin{array}{l}\text { Frecuencia } \\
\text { Hace más de }\end{array}$ & Total & Hombres & Mujeres \\
\hline $\begin{array}{l}\text { un año } \\
\text { ¿Qué tan seguido te haz lastimado en el último mes? }\end{array}$ \\
\hline $\begin{array}{l}\text { 0 veces } \\
\text { 1-3 veces }\end{array}$ & $54.1 \%$ & $18.91 \%$ & $35.13 \%$ \\
\hline $\begin{array}{l}\text { 4-6 veces } \\
\text { Más de 6 } \\
\text { veces }\end{array}$ & $30.8 \%$ & $14.59 \%$ & $16.21 \%$ \\
\hline
\end{tabular}

¿Qué tan seguido te has lastimado en los últimos seis meses?

\begin{tabular}{lccc}
\hline 0 veces & $53 \%$ & $18.91 \%$ & $34 \%$ \\
\hline $1-3$ veces & $27.6 \%$ & $14.1 \%$ & $13.5 \%$ \\
\hline $\begin{array}{l}4-6 \text { veces } \\
\begin{array}{l}\text { Más de } 6 \\
\text { veces }\end{array}\end{array}$ & $14.1 \%$ & $3.78 \%$ & $10.27 \%$ \\
\hline
\end{tabular}

¿Qué tan seguido te has lastimado en el último año?

\begin{tabular}{lccc}
0 veces & $53.5 \%$ & $18.91 \%$ & $34 \%$ \\
\hline 5 veces & $25.9 \%$ & $10.81 \%$ & $15.13 \%$ \\
$\begin{array}{l}\text { Más de } 5 \\
\text { veces }\end{array}$ & $20.5 \%$ & $9.18 \%$ & $11.35 \%$ \\
\hline
\end{tabular}

\section{Modo de autolesión}

Para explorar los modos de autolesión utilizados se proporcionaron cuatro opciones de respuesta: cortadas, marcarse o rayarse, rascar cicatrices y golpeándose (indicando una o varias según correspondiera), y se dejó espacio para escribir modos de lesión. En la tabla 3 se muestra la frecuencia de cada modo de autolesión y la cantidad de maneras de autolesión por caso. Además de las opciones propuestas hubo dos estudiantes que reportaron haberse lastimado ingiriendo fármacos; otro que reportó lastimarse por quemaduras y otro contestó haber tenido un intento de suicidio.

Tabla 3.

Prevalencia de autolesión.

\begin{tabular}{|lcc|}
\hline \multicolumn{3}{c}{ Modo de Autolesión (N=185) } \\
\hline Tipo de Autolesión & FreCUenCia & PorCENTAJE \\
\hline Cortadas & 114 & $61.62 \%$ \\
\hline Marcarse o rayarse & 65 & $35.10 \%$ \\
\hline Rascar cicatrices & 38 & $20.54 \%$ \\
\hline Golpeándose & 61 & $32.90 \%$ \\
\hline
\end{tabular}

\begin{tabular}{|c|c|c|}
\hline \multicolumn{3}{|c|}{$\begin{array}{l}\text { NúMERO dE MOdOS DE AUTOLESIÓN EMPLEAdOS POR CADA } \\
\text { ALUMNO (185) }\end{array}$} \\
\hline TIPO DE AUTOLESIÓN & Frecuencia & Porcentaje \\
\hline 1 modo & 114 & $61.62 \%$ \\
\hline 2 modos & 49 & $26.40 \%$ \\
\hline 3 modos & 13 & $7.02 \%$ \\
\hline 4 modos & 8 & $4.30 \%$ \\
\hline 5 modos & 1 & $0.50 \%$ \\
\hline
\end{tabular}

\section{Motivo de autolesión}

Para indagar acerca de los diferentes motivos para autolesionarse se proporcionaron varias opciones de respuesta a elegir (una o varias, según correspondiera), y se dejó un espacio de "otros motivos" para que los participantes escribieran otra opción (tabla 4).

\section{Tabla 4.}

Motivos de autolesión.

\begin{tabular}{|lcc|}
\hline \multicolumn{1}{c}{ Motivo } & FreCUENCIA & PORCENTAJE \\
\hline Por tristeza & 94 & 50.81 \\
\hline Tratar de sentirme mejor & 80 & 43.24 \\
\hline Por enojo & 71 & 38.37 \\
\hline Por ansiedad/nervios & 47 & 25.40 \\
\hline $\begin{array}{l}\text { Distaerme de lo que me está } \\
\text { molestando }\end{array}$ & 36 & 19.45 \\
\hline $\begin{array}{l}\text { Por soledad } \\
\text { Por experimentación/saber }\end{array}$ & 34 & 18.37 \\
\hline que se sentía & 32 & 17.29 \\
\hline Llamar la atención & 9 & 4.86 \\
\hline $\begin{array}{l}\text { Por presión de compañeros } \\
\text { Otros motivos: Culpa, } \\
\text { problemas familiares }\end{array}$ & 7 & 3.78 \\
\hline
\end{tabular}

Se encontraron diferencias por sexo estadísticamente significativas de cada una de las variables en la muestra total; sin embargo, en la muestra de autolesión sólo hubo diferencias estadísticamente significativas en tres ítems: haber pensado lastimarse, haberse autolesionado al menos una vez y haber visto a alguien más que lo hiciera.

Un aspecto importante a considerar es la planeación de iniciar la conducta de autolesión (los dos ítems que corresponden a este aspecto quedaron fuera la estructura factorial del instrumento); hubo 444 estudiantes que refirieron no haberse lastimado a sí mismos; sin embargo, 66 (14.86\%) contestaron sí haber pensado lastimarse a sí mismos (5.85\% hombres y 9\% mujeres) y 112 
$(25.25 \%)$ respondieron ya haber intentado lastimarse alguna vez (10.58\% hombres y $14.63 \%$ mujeres).

Se preguntó la edad en que sucedió el primer episodio de autolesión; el rango de respuestas fue de los siete a los 14 años, siendo la media de 11.18 (DE $=1.32$ ). A pesar de que el promedio de inicio de autolesión es consistente con lo reportado en otras investigaciones (Vega, Sintes, Fernández, Punti, Soler, Santamarina y Pascual, 2018), se encontró un índice considerable de adolescentes que comenzaron a lesionarse en la infancia (7-10 años), por lo cual es necesario indagar más acerca de la incidencia a edades tempranas, tal como se ha indicado en algunos estudios (Whitlock, Eckenrode \& Silverman, 2006).

EI DSM-5 (APA, 2013) muestra como criterio diagnóstico para la autolesión no suicida haberse hecho lesiones con daño físico leve o moderado al menos cinco días del último año; de acuerdo con ello, 86 adolescentes $(13.67 \%$ de la muestra total) cumplieron con dicho criterio; aún si no se consideran los demás criterios diagnósticos, es un índice importante. Por otro lado, es necesario indicar que $99(15.73 \%)$ reportaron haberse lastimado, pero menos de cinco veces, en el último año; un índice aún mayor que sí se lesiona, pero con menor frecuencia.

De los adolescentes que reportaron lesionarse, 60 $(32.4 \%)$ respondieron haberlo hecho con la intención de quitarse la vida al menos una vez, mientras que 17 (9.1\%) respondieron que lo hicieron con frecuencia o siempre con esa intención. Se requiere especial atención con esta población en la que se refleja una clara relación entre autolesión y suicidio (Vega et al., 2018), aun cuando debe recordarse que en las entrevistas se detectaron antecedentes de intento de suicidio; sin embargo, la mayoría entrevistada respondió no haberlo intentado; lo que refirieron más bien fue herirse y tener pensamientos o ideación suicida, no llegando al punto de intentar el suicidio.

\section{Discusión}

El objetivo de este estudio fue desarrollar un instrumento válido y confiable que evaluara variables relacionadas con autolesión poco estudiadas. Se siguió un cuidadoso procedimiento metodológico buscando incrementar las propiedades psicométricas del mismo, con laboratorios cognoscitivos, validez de contenido por jueceo y entrevistas, y validez de constructo mediante el AFE y el AFC.

La validez de contenido fue adecuada según los altos valores del coeficiente kappa obtenidos por acuerdos de los jueces, así como por la congruencia entrevista/cuestionario, aunque se encontró un pequeño índice de inconsistencia en las variables relacionadas con la frecuencia de autolesión en diferentes periodos. Sin em- bargo, el problema de inconsistencia podría deberse al tipo de respuestas que se solicita al participante y que implica la realización simultánea de dos diferentes actividades, recordar la cantidad de eventos y estimar los periodos en que ocurrieron, pues como indican Streiner, Norman \& Cairney (2015), los ítems con doble tarea generan más dificultades en sus respuestas.

Los factoriales produjeron resultados teórica y estadísticamente valiosos; los tres factores resultantes no sólo explicaron altos índices de varianza; también mostraron buena confiabilidad o consistencia interna. Lo importante a destacar es que el AFC no sólo replicó la estructura del AFE, sino que también mejoró la magnitud de las cargas factoriales. Es interesante indicar la asociación entre la frecuencia de autolesión y su efecto adictivo, por lo que no deja de llamar la atención que una parte importante de los participantes que reportaron dificultad para dejar de lastimarse hayan tenido uno o más episodios de autolesión durante el último mes.

El primer factor proporciona información de la cantidad de lesiones en diferentes periodos, permitiendo tener una estimación aproximada del desarrollo de la autolesión a lo largo del tiempo, e incluso, como se comprobó con las entrevistas, se pueden contextualizar y comprender con más claridad los hechos desencadenantes y/o los efectos que mantienen dicha conducta.

El segundo factor, la dificultad para dejar de lastimarse, es un aspecto importante en el desarrollo de la autolesión, precisamente la cronicidad con que se hacen las heridas es lo que puede definir la gravedad del caso, junto con la presencia de un mayor repertorio de formas de dañarse y el riesgo de ideación suicida (Hamza, Stewart \& Willoughby, 2012). Este aspecto, y la frecuencia de autolesión, proporcionan datos importantes para la detección de casos con autolesión de mayor gravedad.

El tercer factor tiene ítems referentes al contagio social; la información que puede derivarse de este factor es de gran importancia para efectos de detección temprana y prevención, y debe destacarse que aquí se propone por primera vez, en nuestro contexto social, el estudio de la relación de esta variable; no debe olvidarse que los medios de comunicación y la influencia de los pares pueden ser determinantes en el desarrollo de la autolesión (Purington \& Whitlock, 2010).

En cuanto a la frecuencia de la autolesión se obtuvieron resultados similares a los hallados por Albores-Gallo et al. (2014) respecto al último mes, tres meses y el último año; sin embargo, hubo una gran diferencia acerca del índice de participantes que reportó haberse lastimado al menos una vez (sin especificar un periodo); 
en este estudio se encontró $29.4 \%$, casi el doble que en el estudio mencionado.

Algunas formas de autolesión (como cortarse o golpearse a sí mismo) se encontraron con una alta frecuencia, de manera similar a otros estudios (Briere \& Gil, 1998; Nock, 2010); sin embargo, otras formas de lastimarse que suelen hallarse, aunque con menor frecuencia, en este estudio casi no se reportaron, aun cuando se daba opción a escribir con confianza diferentes formas de lastimarse.

Los motivos para lesionarse que reportaron los participantes permiten observar que la autolesión está, en la mayoría de los casos, relacionada con las emociones; en las entrevistas constantemente se hizo alusión a las autolesiones como un desahogo, una forma de expresarse y sentirse mejor. Es interesante notar que son diferentes emociones las que pueden influir en el origen de las autolesiones, coincidiendo con los hallazgos que indican un rol fundamental de la regulación emocional en las autolesiones (Klonsky, 2009).

\section{Conclusiones}

Los resultados descritos aportan evidencias preliminares de validez y confiabilidad del cuestionario riesgo de autolesión, así como información objetiva y valiosa para la elaboración de un nuevo instrumento útil para evaluar la autolesión en cuanto a su frecuencia, su efecto adictivo y contagio social en adolescentes de 11 a 15 años. La detección temprana de jóvenes con autolesión puede ser fundamental para proporcionarles el tratamiento adecuado, evitando así que los episodios de autolesión se tornen frecuentes, generando cuadros clínicos con niveles psicopatológicos, además de que se podría disminuir, e idealmente eliminar, la propagación de la autolesión.

Los hallazgos de este estudio proponen ahondar en aspectos poco analizados antes por otros instrumentos, como el componente adictivo y el contagio social, de los cuales se presenta una opción válida y confiable; también se propuso analizar la intención de autolesionarse; sin embargo es necesaria más investigación en dicho aspecto, porque podría ser un determinante en la prevención temprana.

\section{Limitaciones y sugerencias}

Las limitaciones son las inherentes a los estudios preliminares; algunos aspectos evaluados tienen antecedentes teóricos, pero pocos estudios empíricos con los cuales compararlos. Los resultados encontrados en la muestra no se pueden generalizar a la población de donde se extrajo dicha muestra porque la selección no fue aleatoria. Son necesarias nuevas investigaciones incrementando el conocimiento mediante el uso de nuevas y más variables o ítems y, entre otras cosas, replicando el presente estudio en muestras de otras poblaciones; por ejemplo, con un mayor rango de edad, considerando que la autolesión mantiene una alta prevalencia en adolescentes y adultos jóvenes (Vega et al., 2018), y con procedimientos metodológicos y estadísticos más estrictos.

Se sugiere la aplicación de entrevistas porque enriquecen la información obtenida por medio de los cuestionarios; en este estudio sirvieron para comparar las respuestas obtenidas, detectar tendencias en las problemáticas y experiencias de diferentes casos, y para detectar adolescentes con alto riesgo, quienes requirieron canalización inmediata a centros de atención psicológica (el análisis completo no se proporciona aquí porque el propósito de este estudio fue el desarrollo de las propiedades psicométricas del instrumento y no el análisis cualitativo de los resultados del mismo).

\section{Referencias}

Albores-Gallo, L., Méndez-Santos, J. L., García, L. A. X., DelgadilloGonzález, Y., Chávez, C. I., \& Martínez, O. L. (2014). Autolesiones sin intención suicida en una muestra de niños y adolescentes de la Ciudad de México. Actas Españolas de Psiquiatría, 42(4), 159-168.

American Psychiatric Association (APA) (2013). Diagnostic and statistical manual of mental disorders (5a. ed.). Washington, DC, USA: autor.

Bartlett, M. S. (1950). Tests of significance in factor analysis. British Journal of Psychology, 3, 77-85.

Briere, J., \& Gil, E. (1998). Self mutilation in clinical and general population samples: Prevalence, Correlates, and Functions. American Journal of Orthopsychiatry, 68, 609620. doi: $10.1037 / \mathrm{h} 0080369$.

Cipriano, A., Cella, S., \& Cotrufo, P. (2017). Nonsuicidal Selfinjury: A Systematic Review. Frontiers in Psychology, 8, 1-14. doi: 10.3389/fpsyg.2017.01946.

Costello, A. B., \& Osborne, J. W. (2005). Best practices in exploratory factor analysis: Four recommendations for getting the most from your analysis. Practical Assessment, Research, \& Evaluation, 10,(7), 1-9. Recuperado de: http:// pareonline.net/genpare.asp?wh=0\&abt=10

Eisenkraft, M. A. (2006). Self Injury: Is It a Syndrome? The New School Psychology Bulletin, 4(1), 115-126. Recuperado de: http://www.columbia.edu/ me2357/shp/29-108-1-PB-1.pdf

Favazza, A. (1996). Bodies under siege. Baltimore: John Hopkins University Press.

Farber, S. K., Jackson, C. C., Tabin, J. K., \& Bachar, E. (2007). Death and annihilation anxieties in anorexia nervosa, bulimia, and self-mutilation. Psychoanalytic Psychology, 24(2), 289-305. doi: 10.1037/0736-9735.24.2.289.

Fleiss, J. L. (1971). Measuring nominal scale agreement among many raters. Psychological Bulletin, 76(5), 378-382. doi: 10.1037/h0031619. 
Flores, Y.V.C., Hidalgo-Rasmussen, C. A., \& Peñúñuri, L.Y.Y.(2017). Instrumentos de evaluación de la autolesión no suicida en adolescentes 1990-2016: Una revisión sistemática. Ciencia \& saúde colectiva. Recuperado de: http://www. cienciaesaudecoletiva.com.br/artigos/instrumentos-deevaluacion-de-la-autolesion-no-suicida-en-adolescentes19902016-una-revision-sistematica/16533?id=16533

Gandhi, A., Luyckx, K., Baetens, I., Kiekens, G., Sleuwaegen, E., Berens, A., \& Claes, L. (2017). Age of onset of nonsuicidal self-injury in Dutch-speaking adolescents and emerging adults: An event history analysis of pooled data. Comprehensive psychiatry, 80, 170-178. doi: 10.1016/j. comppsych.2017.10.007.

Gratz, K. (2001). Measurement of deliberate self-harm: preliminary data on the deliberate self-harm Inventory. Journal of Psychopathology and Behavioral Assessment, 23, 253-263. doi: 10.1023/A:1012779403943.

Hamza, C. A., Stewart, S. L., \& Willoughby, T. (2012). Examining the link between nonsuicidal self-injury and suicidal behavior: A review of the literature and an integrated model. Clinical Psychology Review, 32, 482-495. doi: 10.1016/j.cpr.2012.05.003.

Hu, L., \& Bentler, P. (1999). Cutoff criteria for fit indexes in covariance structure analysis: Conventional criteria versus new alternatives. Structural Equation Modeling: A Multidisciplinary Journal, 6, 1-55. doi: 10.1080/10705519909540118.

Kaiser, H. F. (1974). An index of factorial simplicity. Psychometrika, 39, 31-36. doi: 10.1007/BF02291575.

Kim, J. O., \& Mueller, C. W. (1978). Factor analysis: Statistical methods and practical issues. Sage University Paper Series on Quantitative Applications n the Social Sciences, series no. 07-014. Newbury Park, CA: Sage.

Kline, R. B. (2005). Principles and practice of structural equating modeling. Nueva York: The Guilford Press.

Klonsky, E. D. (2009). The functions of self-injury in young adults who cut themselves: Clarifying the evidence for affect-regulation. Psychiatry Research, 166(2-3), 260-268. doi:10.1016/j.psychres.2008.02.008.

Lloret-Segura, S., Ferreres-Traver, A., Hernández-Baeza, A., \& Tomás-Marco, I. (2014). El análisis factorial exploratorio de los ítems: Una guía práctica, revisada y actualizada. Anales de Psicología, 30(3), 1151-1169. doi: 10.6018/ analesps.30.3.199361.

Lorenzo-Seva, U. (1999). Promin: A method for oblique factor rotation. Multivariate Behavioral Research, 34, 347-356. doi: 10.1207/S15327906MBR3403_3.

Lorenzo-Seva, U., \& Ferrando, P. (2007). FACTOR: A computer program to fit the exploratory factor analysis model. Behavior research methods, 38(1), 88-91.

MacCallum, R. C., Widaman, K. F., Zhang, S., \& Hong, S. (1999). Sample size in factor analysis. Psychological Methods, 4, 84-99. doi:10.1037/1082-989X.4.1.

Mangall, J., \& Yurkovich, E. (2008). A literature review of deliberate self-harm. Perspectives in psychiatric care, 44(3), 175-184. doi: 10.1111/j.1744-6163.2008.00172.x.
Marín, T. M. I. (2013). Desarrollo y evaluación de un tratamiento cognitivo conductual para adolescentes que se autolesionan. Tesis doctoral. Facultad de Psicología. México: Univesidad Nacional Autónoma de México.

Nock, M. K. (2010). Self-Injury. Annual Review of Clinical Psychology, 6(1), 339-63. doi: 10.1146/annurev. clinpsy.121208.131258.

Nunnally, J. C. (1978). Psychometric theory (2a. ed.). Nueva York: McGraw-Hill.

Pattison, E. M., \& Kahan, J. (1983). The deliberate self-harm syndrome. American Journal of Psychiatry, 140, 867-872. doi:10.1176/ajp.140.7.867.

Purington, A., \& Whitlock, J. (2010). Non-suicidal self-injury in the media. The Prevention Researcher, 17(1), 11-13. Recuperado de: http://www.selfinjury.bctr.cornell.edu/ perch/resources/non-suicidal-self-injury-in-the-media.pdf

Santos, B. D. (2011). Autolesión: Qué es y cómo ayudar. México: Ficticia.

Satorra, A., \& Bentler, P. M. (1988). Scaling corrections for statistics in covariance structure analysis. UCLA Statistics Series \#2. Los Ángeles: University of California.

Satorra, A., \& Bentler, P. M. (1994). Corrections to test statistics and standard errors in covariance structure analysis. In A. Von Eye \& C. C. Clogg (eds.). Latent variables analysis: Applications for developmental research (pp. 399-419). Thousand Oaks: Sage.

Sociedad Mexicana de Psicología (SMP) (2007). Código ético del psicólogo (4a. ed.). México: Trillas.

Steiger, J. H. (2007). Understanding the limitations of global fit assessment in structural equation modeling. Personality and Individual Differences, 42(5), 893-98. doi: 10.1016/j. paid.2006.09.017.

Streiner, D., Norman, G., \& Cairney,J. (2015). Health Measurement Scales. A practical guide to their development and use. UK: Oxford University Press.

Vega, D., Sintes, A., Fernández, M., Punti, J., Soler, J., Santamarina, P., \& Pascual, J. C. (2018). Revisión y actualización de la autolesión no suicida: ¿Quién, cómo y por qué? Actas españolas de psiquiatría, 46(4), 146-155. Recuperado de: https://www.actaspsiquiatria.es/repositorio/20/114/ ESP/20-114-ESP-146-55-463018.pdf

Velicer, W. F., \& Fava, J. L. (1998). Effects of variable and subject sampling on factor pattern recovery. Psychological Methods, 3, 231-251. doi: 10.1037//1082-989X.3.2.231.

Walsh, W. B. (2006). Treating self-injury. A practical guide. Nueva York: The Guilford Press.

Whitlock, J., Eckenrode, J., \& Silverman, D. (2006). Self-injurious behaviors in a college population. Pediatrics. 117(6) 193948. doi: $10.1542 /$ peds.2005-2543.

Williams, B., Brown, T., \& Onsman, A. (2010). Exploratory factor analysis: A five-step guide for novices. Australasian Journal of Paramedicine, 8(3), 1-13. doi:10.33151/ajp.8.3.93. 


\section{Meta-Análisis del Artículo}




\section{Dimensión Cuantitativa}

\section{Perfil de Evaluación entre pares}
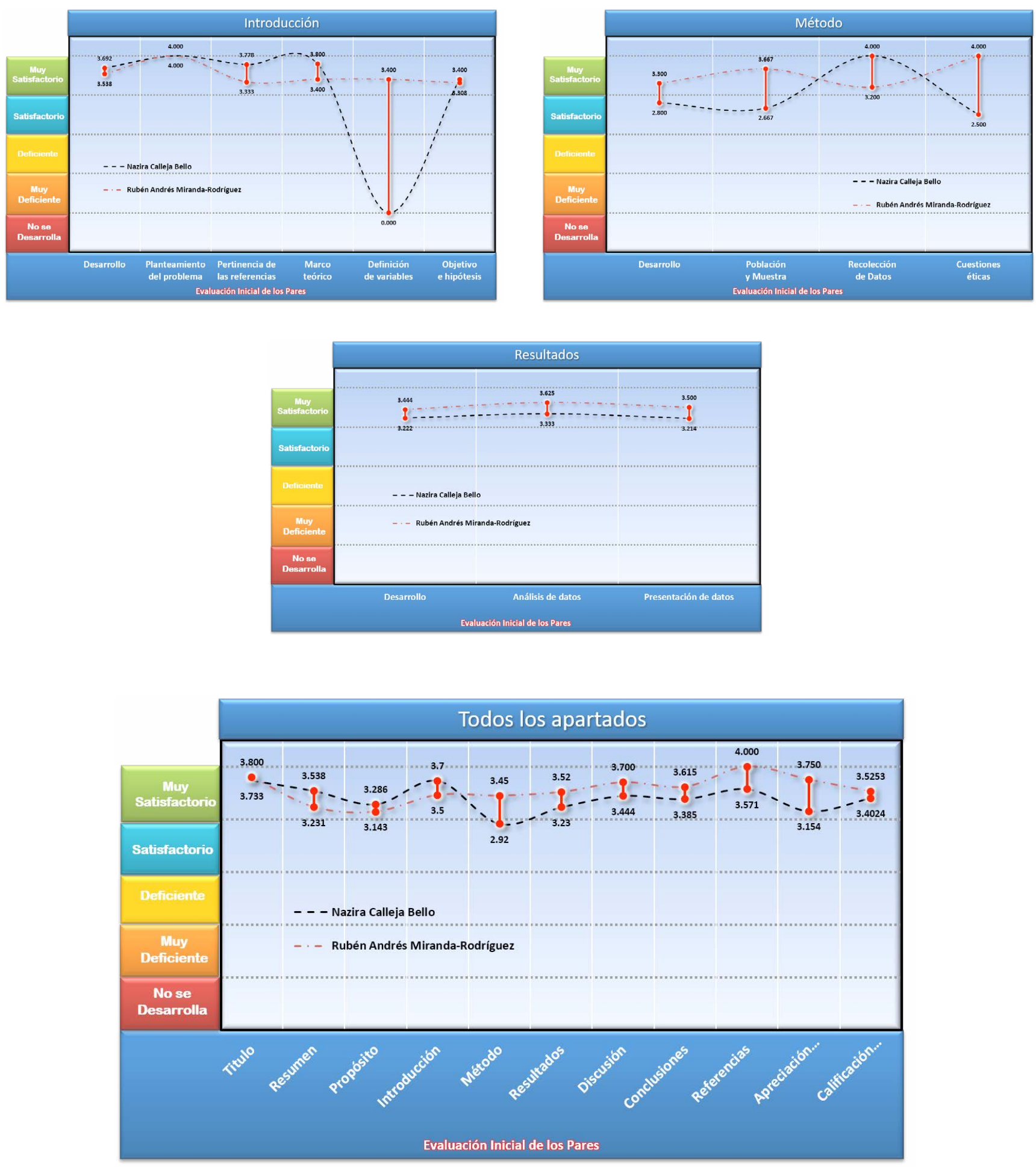


\section{Índice de Concordancia}

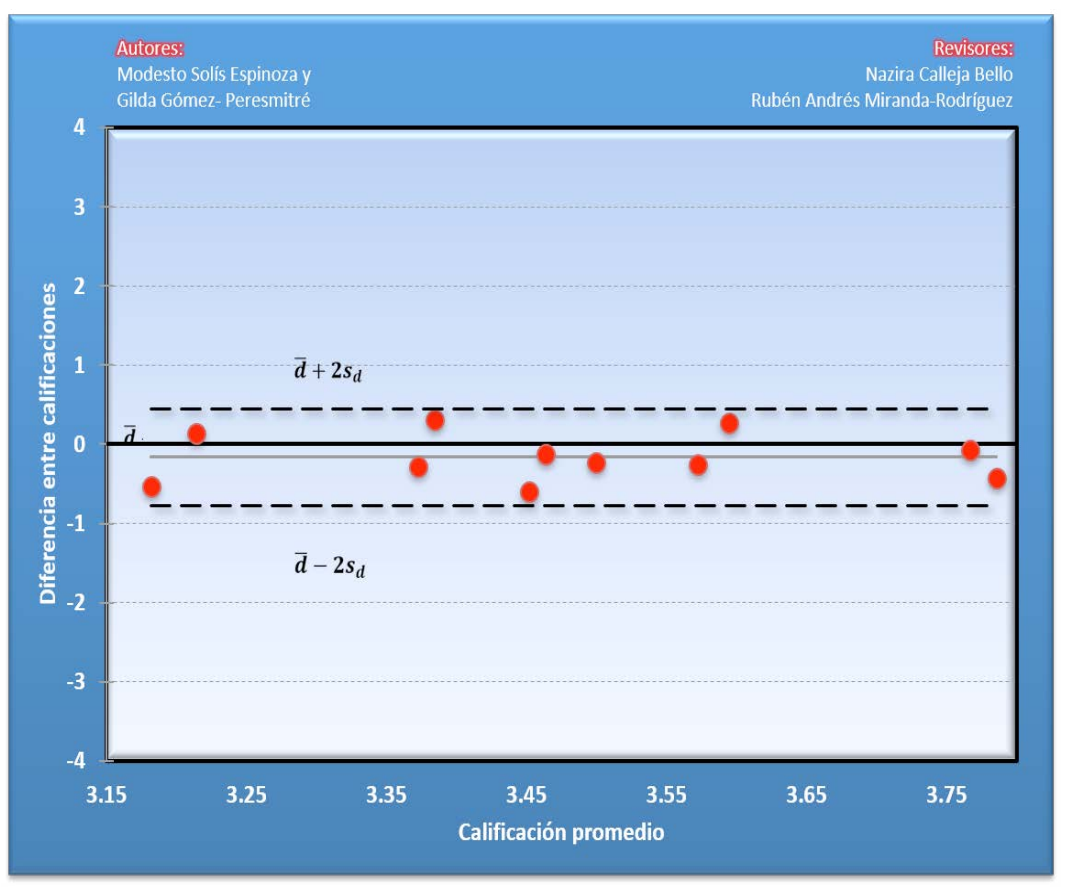

Índice de Acuerdo

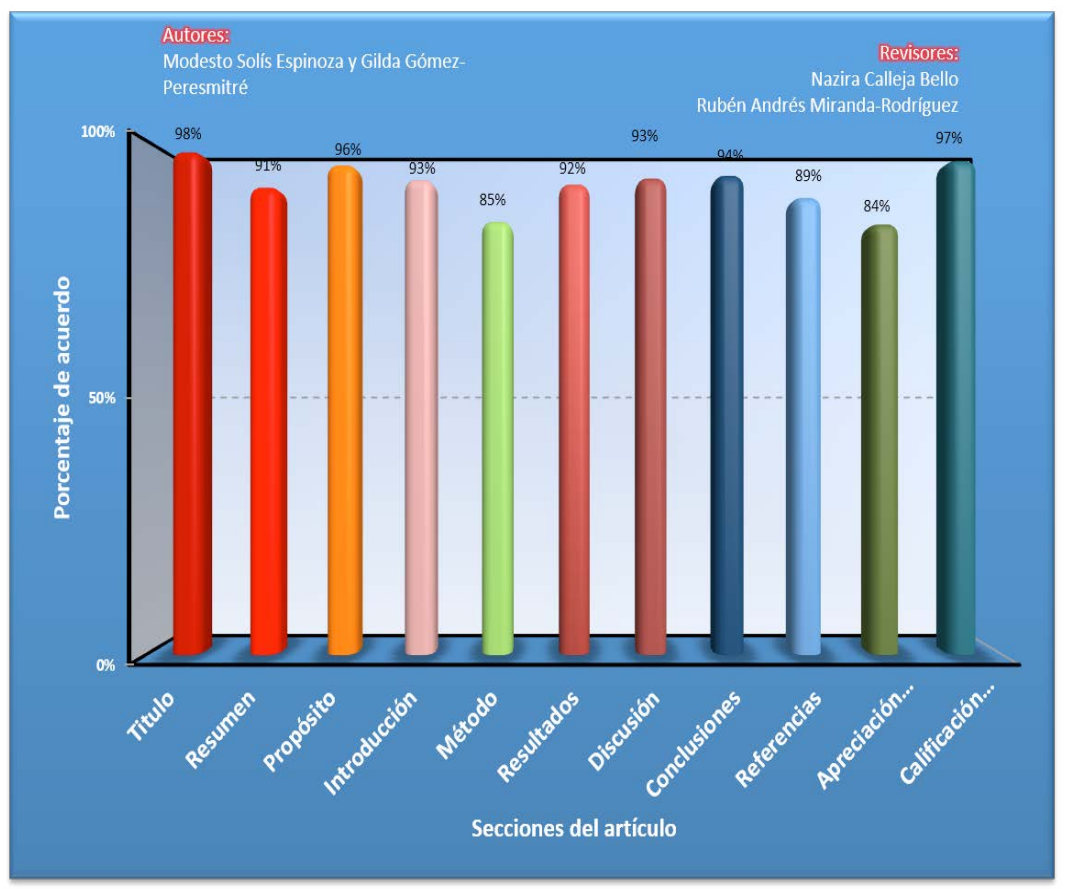




\begin{tabular}{|c|c|}
\hline Revisor 1 & Revisor 2 \\
\hline Nazira Calleja Bello & Rubén Andrés Miranda Rodríguez \\
\hline
\end{tabular}

Título/Autoría

El instrumento desarrollado consta de tres dimensiones: frecuencia de autolesión, adicción y contagio. De ellas, sólo la tercera puede ser considerada propiamente "riesgo", por lo que quizá “...un instrumento que mide riesgo de autolesión" no sea la denominación más apropiada. Se sugiere, además que, en lugar de "un instrumento que mide..., señalar el nombre específico que se ha dado al instrumento (Cuestionario de Riesgo de Autolesión, CRA), e incluir las palabras "en adolescentes". Además del desarrollo y validación del instrumento, el estudio incluye también resultados en relación con la frecuencia, modo y motivo de autolesión, la planeación de iniciar la conducta de autolesión y la edad en que sucedió el primer episodio, y efectúa comparaciones por sexo, por lo que convendría hacer alguna mención al respecto en el título.

Se considera apropiado.

\section{Resumen}

a) Se sugiere revisar la redacción (puntuación, uso de mayúsculas, siglas, espacios, así como hacer corresponder el resumen y el abstract. b) Dice: “... que explorara la presencia de autolesión", pero se evaluó la frecuencia, no sólo la presencia. c) ¿"Dificultad para dejar de lesionarse" realmente es lo mismo que "efecto adictivo"? d) Falta incluir las conclusiones del estudio respecto del objetivo planteado.

Tiene 197 palabras. Se recomienda reducirlo.

\section{Próposito del Estudio}

Se efectuaron comparaciones por sexo que no están propuestas en los objetivos del estudio. Tampoco lo están los análisis de frecuencia, modo y motivo de autolesión, la planeación de iniciar la conducta de autolesión, la edad en que sucedió el primer episodio y la intención de quitarse la vida. Éstos se presentan como resultados complementarios, pero en realidad son parte esencial del estudio, por lo que deberían incorporarse a los propósitos de la investigación.

Se considera claro y satisfactorio. 


\section{Revisor 1}

Revisor 2

\section{Introducción}

Sugerencias y preguntas: a) Señalar la(s) citas(s) específicas en las que se basa la definición de autolesión. b) Dice “...de naturaleza socialmente inaceptable"; valdría la pena analizar si esto es así en el caso de los adolescentes, considerando, además, que se está evaluando una dimensión de contagio social. c) En el primer párrafo se indica que "...sucede la mayoría de las veces en ausencia de psicosis..., pero en el párrafo dos se afirma que "...hasta un $50 \%$ de los que se producen autolesión pueden padecer un trastorno mental". ¿En estos casos se trata de autolesión psicótica? d) ¿Cuales serán "otros comportamientos autodestructivos"? e) Se sugiere ampliar la información de otros instrumentos previamente desarrollados para medir autolesión, incluir la información psicométrica de aquellos que la hayan reportado, indicar sus ventajas y desventajas, y señalar claramente las razones por las que se decisión desarrollar uno instrumento más. f) Se efectuaron comparaciones por sexo que no están propuestas en los objetivos del estudio. Tampoco lo están los análisis de frecuencia, modo y motivo de autolesión, la planeación de iniciar la conducta de autolesión, la edad en que sucedió el primer episodio y la intención de quitarse la vida. Éstos se presentan como resultados complementarios, pero en realidad son parte esencial del estudio. e) Revisar la redacción de la introducción.

Se considera adecuada.

\section{Método}

Sugerencias y preguntas a) Describir más ampliamente las características de los participantes (no sólo su sexo y edad). b) Señalar la razón de que se haya obtenido, a partir de la muestra total, una submuestra $(n=241)$, e indicar el procedimiento de selección. ¿Con esta muestra se efectuaron todos los procedimientos? c) ¿Qué ocurrió con los reactivos elaborados para explorar la presencia de autolesión y la intención de realizarla? d) ¿Cuáles fueron las opciones de respuesta? ¿Por qué unos reactivos fueron dicotómicos y otros politómicos? ¿Por qué se decidió "igualar todas las variables a nivel de medición dicotómica", teniendo reactivos con siente opciones de respuesta? ¿Todos los procedimientos psicométricos (AFE, AFC, alfa, omega) se efectuaron con sólo dos opciones de respuesta? e) Describir el procedimiento de validación de contenido: núSe recomienda justificar por qué se utilizó la misma muestra tanto para Análisis Factorial Exploratorio como Análisis Factorial Confirmatorio. mero y características de los jueces, opciones de jueceo. ¿Por qué se eligió el índice Kappa? f) Describir el procedimiento y los resultados de los laboratorios cognoscitivos: número de laboratorio, número y características de los participantes, instrucciones, lugar. g) En la sección de Procedimiento se indica que "se entrevistó a un(?) $20 \%$ de los participantes para explorar la concordancia.... ¿Cuáles fueron las preguntas de la entrevista? ¿Cómo se exploró la concordancia? h) ¿Por qué en la sección de Mediciones se presenta la definición del constructo? i) Revisar la redacción. 


\section{Revisor 1}

Revisor 2

Resultados

Sugerencias y preguntas: a) Ampliar los resultados de la validación de contenido por jueces. ¿Qué decisiones se tomaron a partir de ellos? No se indican los IC. Evitar juicios de valor ("resultó bueno"). b) Respecto de las respuestas obtenidas en la entrevista, ¿con base en qué se afirma que fueron consistentes con las del cuestionario? ¿Qué es un "bajo porcentaje con respuestas contradictorias"? ¿Cuáles fueron las contradicciones obtenidas? ¿Se obtuvo algún índice de concordancia? c) Dado el contenido de los reactivos, es de suponer que los análisis factoriales se efectuaron sólo con los 185 participantes que reportaron haberse autolesionado. ¿Así fue? Habría que explicitarlo. d) ¿Un $\mathrm{KMO}=0.82$ es "alto"? e) ¿Cuáles fueron los cuatro reactivos eliminados? ¿Por qué resultaron eliminados? f) Los reactivos 3 y 9 son preguntas filtro, cuya respuesta es dicotómica. ¿Realmente deberían estar ubicadas en el factor Efecto (¿o Componente?) Adictivo? g) Revisar el factor Frecuencia, ya que las altas cargas factoriales sugieren autocorrelación, que pueden tener efectos en los altos índices de consistencia interna obtenidos. h) Rotular las tablas adecuadamente. i) Incluir medidas descriptivas de cada factor y las correlaciones inter-factor. j) Se indica que se ensayaron "...varios ajustes a las soluciones factoriales”. ¿Cuáles fueron éstos? k) En la sección de Análisis estadísticos se señala que se tomarían como índices de bondad de ajuste: $\chi^{2}$ / gl, CFI, SRMR y RMSEA. En Resultados se reportaron, además, GFI e IFI, y no SRMR; también faltó el IC para RMSEA. Estos índices están tanto en el texto como en la figura 1.l) Rotular correctamente la figura. Los reactivos aparecen abreviados y sin número. $\mathrm{m}$ ) La carga factorial del reactivo "Sugerencia autolesión" es muy baja (.321). ¿Es posible que se haya copiado incorrectamente? n) ¿Por qué en el apartado de AFC se incluye la prevalencia y frecuencia de autolesión? o) Describir los hallazgos respecto de la frecuencia de autolesión total y por sexo. p) En la tabla 2 podrían eliminarse las frecuencias absolutas y conservar las relativas (\%).Comentar en el texto los resultados principales. q) En la tabla 3, no queda claro a qué corresponde los porcentajes de modo de autolesión. ¿Qué significa "por cada caso"? Comentar en el texto los resultados principales. r) La pregunta relativa al motivo de la autolesión no se incluye en la descripción del instrumento. s) En la tabla 4 , ¿las cantidades corresponden a menciones? Comentar en el texto los resultados principales. t) ¿Por qué se incluye una sección de Resultados complementarios? Habría que integrarlos. u) Se señala que se efectuaron comparaciones por sexo, pero no se incluyen los datos obtenidos. ¿Se realizaron estas comparaciones por reactivo o por factor? v) En la sección de Resultados complementarios se mezclan los datos con la discusión de éstos. w) Revisar la redacción.

Se recomienda aclarar qué método de extracción se utilizó en el análisis factorial exploratorio y por qué fue mejor hacer dicotómicos todos los reactivos en lugar de politómicos. 


\begin{tabular}{|c|c|}
\hline Revisor 1 & Revisor 2 \\
\hline \multicolumn{2}{|c|}{ Resultados } \\
\hline 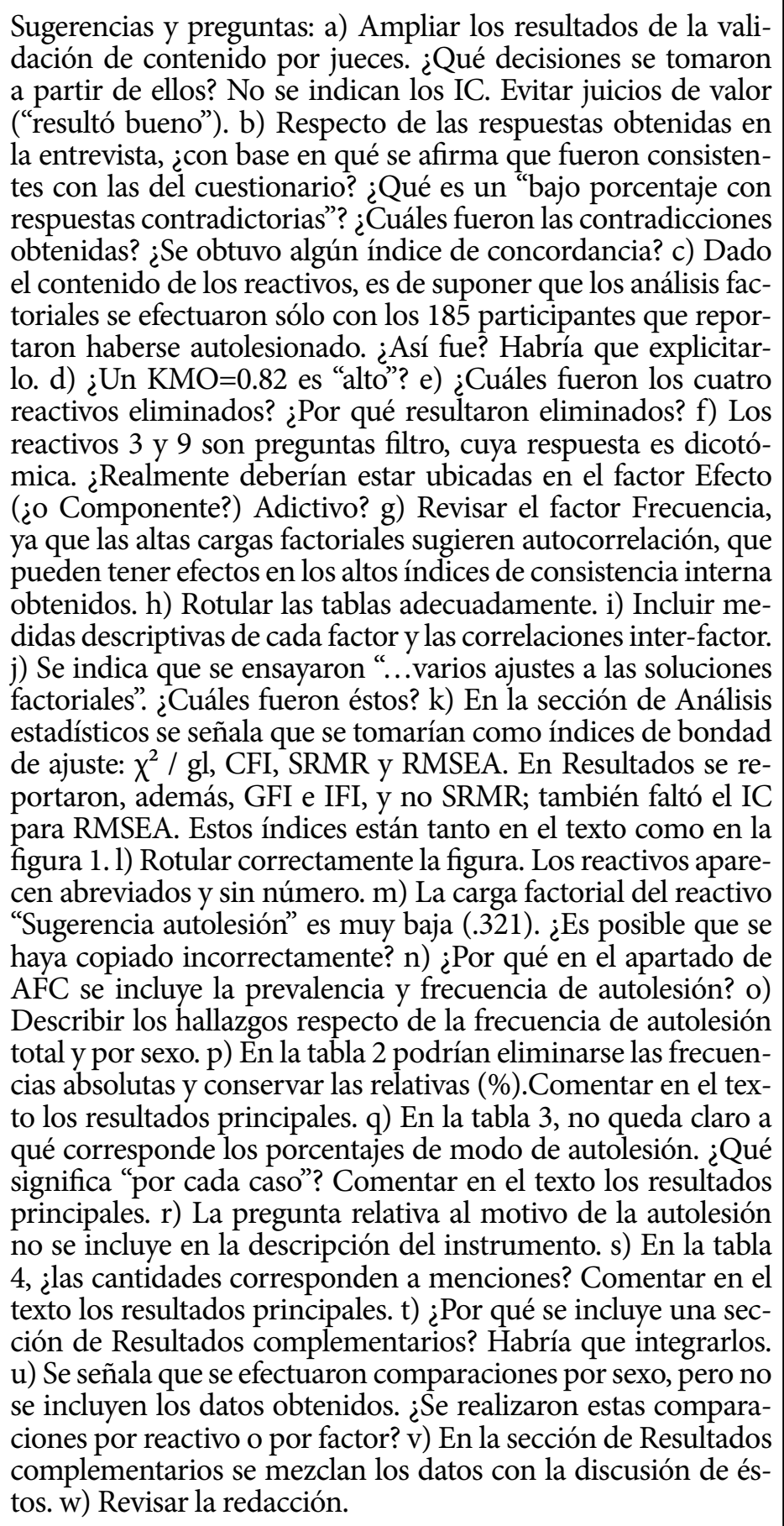 & $\begin{array}{l}\text { Se recomienda aclarar qué método de extracción se utilizó en el } \\
\text { análisis factorial exploratorio y por qué fue mejor hacer dicotó- } \\
\text { micos todos los reactivos en lugar de politómicos. }\end{array}$ \\
\hline
\end{tabular}




\section{Revisor 1}

Revisor 2

\section{Discusión}

Sugerencias: a) Evitar el uso repetido de expresiones como "lo importante", "interesante", "gran relevancia", "no deja de llamar la atención". b) Integrar a la sección de Discusión la interpretación de los datos presentados en Resultados complementarios (preferentemente, eliminar esta sección). c)

Sería plausible indagar en una discusión sobre aspectos psicométricos del constructo. Es decir, el contraste con otras herraUna vez revisada la sección de Resultados, podría enriquemientas de evaluación. cerse la discusión de los hallazgos. d) Revisar la redacción.

\section{Conclusiones}

Se sugiere ampliar las conclusiones, señalando las contribuciones en relación con los instrumentos desarrollados anteriormente.

Se consideran pertinentes.

\section{Referencias}

Se sugiere incluir más referencias de los últimos cinco años.

Cumplen con los requisitos. 
